\title{
The lens of freshwater in a tropical island: the two interface case
}

\author{
S. A. Chen ${ }^{1} \quad$ G. C. Hocking ${ }^{2}$
}

(Received 29 October 2009; revised 10 February 2010)

\begin{abstract}
The lens of freshwater beneath a tropical island is vital for supplying water to the island population. We consider the circumstances under which artificial recharge through a single inlet point will maintain the lens if no natural recharge occurs. A Greens function approach derives an integral equation that is solved numerically for the case in which two interfaces exist - one between salt and freshwater and one between freshwater and air. There appear to be upper and lower bounds on the flow rates that produce steady interface shapes. The height of the seepage faces is dominated by the density ratios.
\end{abstract}

\section{Contents}

1 Introduction

\section{Problem formulation}

http://anziamj . austms.org.au/ojs/index.php/ANZIAMJ/article/view/2599 gives this article, (C) Austral. Mathematical Soc. 2010. Published February 17, 2010. ISSN 1446-8735. (Print two pages per sheet of paper.) 
3 Integral equation for two free surfaces

4 Numerical method

5 Results

6 Concluding remarks

\section{Introduction}

The water supply for many tropical islands comes from a lens of freshwater trapped in the soil beneath the surface and above the saltwater that intrudes from the surrounding ocean; see Figure 1. Langevin et al. [4] and Ruppel et al. [8] discuss the importance of the management of this resource by communities living on small islands. The continued existence of this natural, potable water supply is governed by recharge through rainfall, the location of the interface between the fresh and saltwater layers and the effects of withdrawal. If there is insufficient rainfall, or if too much water is extracted, then the lens may diminish or disappear. To prevent this it is necessary to recharge the lens artificially.

Hocking and Forbes [2], and Forbes et al. [1] computed flows due to withdrawal from the freshwater layer in the two dimensional and axisymmetric cases assuming that the soil was fully saturated within the island. That work includes an implicit assumption that there is sufficient recharge to maintain the water levels no matter how much is withdrawn. The computation of the critical coning behaviour of the interface is analogous to similar problems in unbounded domains $[3,5,6,9$, e.g.].

We consider the case where there is insufficient natural rainfall to recharge the aquifer and artificial pumping is required to replenish it. We allow the 


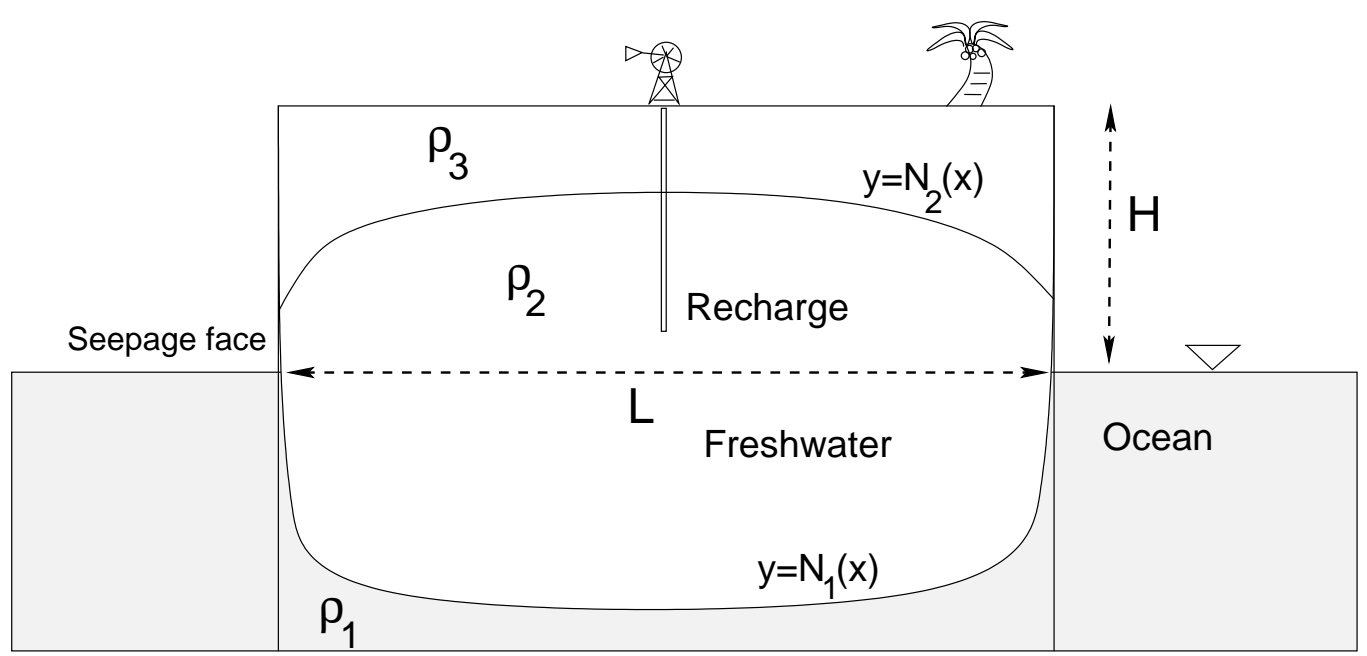

FiguRE 1: Sketch of tropical island of width $L$ and height $H$ with recharge via a line source.

presence of an upper interface between the fresh water layer and the air. This study determines under what conditions the lens is sustainable or can even exist given a single point of influx and no withdrawal.

To obtain a steady flow with inflow and no withdrawal, there must be two seepage faces on the sides of the island. Green's second identity and an appropriate Green's function are used to derive a boundary integral equation for the unknown location of the two interfaces. The output generated gives the shape of two interfaces and the height of the seepage faces $\beta_{ \pm}$after incorporating the relevant parameters, such as density ratios $\gamma_{1}$ and $\gamma_{2}$, inflow rate, $\mu$, and island length, $\alpha$.

Section 2 derives the equations of the problem. Unlike the single free surface case, there is no recharge of fluid through the upper surface of the island. Section 3 formulates the problem as an integral equation using a Green's function. The details of the numerical scheme are given in Section 4 and results and conclusions follow. 


\section{Problem formulation}

Consider three fluids of different density, air, freshwater and saltwater, in a porous medium of length, L, and height, $\mathrm{H}$, above sea level. We choose a Cartesian coordinate system $(x, y)$ centred in the middle of the island at sea level. The seepage velocity vector $\vec{q}_{i}$ in each layer $i=1,2,3$ is given by Darcy's Law as

$$
\overrightarrow{\mathrm{q}}_{i}=-\kappa \nabla\left(p_{i}+\rho_{i} g y\right),
$$

where $k$ is the total permeability of the rock and $p_{i}$ is the pressure. We define the piezometric heads as

$$
\Phi_{i}=p_{i}+\rho_{i} g y, \quad i=1,2,3,
$$

where the subscripts 1,2,3 denote the variables corresponding to the layers of saltwater, freshwater and air, respectively. Assuming that the rock is fully saturated means that the continuity equation is

$$
\nabla \cdot \vec{q}_{i}=0 \quad \text { for } i=1,2,3 .
$$

Noting equation (1) and assuming the value of $\boldsymbol{k}$ to be constant leads to Laplace's equation

$$
\nabla^{2} \Phi_{i}=0, \quad i=1,2,3,
$$

in each of the three layers. Here, the majority of the flow occurs within the freshwater layer, and as a consequence the flow in the air and saltwater layers is assumed to be negligible so that $\Phi_{1}$ and $\Phi_{3}$ are constant. The region of fresh water is then bounded by the lower interface, $y=N_{1}(x)$, between salt and freshwater and the upper interface, $y=N_{2}(x)$, between air and fresh water. Across the two interfaces, the pressures must match, so that

$$
\begin{array}{ll}
\Phi_{2}=\Phi_{1}-\left(\rho_{1}-\rho_{2}\right) N_{1} g & \text { on } y=N_{1}(x), \\
\Phi_{2}=\Phi_{3}-\left(\rho_{3}-\rho_{2}\right) N_{2} g & \text { on } y=N_{2}(x),
\end{array}
$$

and since there is no flow through the two interfaces,

$$
\overrightarrow{\mathrm{q}}_{2} \cdot \overrightarrow{\mathrm{n}}_{\mathrm{k}}=0 \quad \text { on } \mathrm{y}=\mathrm{N}_{\mathrm{k}}(\mathrm{x}), \quad \mathrm{k}=1,2,
$$


where $\vec{n}_{k}$ are the normals to the interfaces, $k=1,2$.

The two seepage faces are in contact with the air, and so we obtain

$$
\Phi_{2}=-\left(\rho_{3}-\rho_{2}\right) g y \quad \text { on } x= \pm L, \quad 0<y<B_{ \pm},
$$

where $B_{ \pm}$refers to the heights of the seepage faces at $x= \pm L / 2$, respectively. Non-dimensionalizing with respect to the island height $\mathrm{H}$, and the middle layer potential $\rho_{2} \mathrm{gH}$, and letting $\rho_{1} / \rho_{2}=\gamma_{1}>1$ and $\rho_{3} / \rho_{2}=\gamma_{2}<1$, gives

$$
\begin{aligned}
& \phi_{2}=\phi_{1}+\left(1-\gamma_{1}\right) \eta_{1} \quad \text { on } y=\eta_{1}(x), \\
& \phi_{2}=\left(1-\gamma_{2}\right) \eta_{2} \quad \text { on } y=\eta_{2}(x), \\
& \phi_{2}=y \quad \text { on } x= \pm \alpha / 2, \quad 0<y<\beta_{ \pm},
\end{aligned}
$$

for the potential $\phi_{2}$ and interfaces $\eta_{1}(x)$ and $\eta_{2}(x)$ on an island of nondimensional width $\alpha=\mathrm{L} / \mathrm{H}$ with seepage faces of height $\beta_{ \pm}=\mathrm{B}_{ \pm} / \mathrm{H}$ at $\mathrm{x}= \pm \alpha / 2$. Without loss of generality we set $\phi_{3}=0$ and $\phi_{1}$ is constant. Henceforth, we drop the subscript ' 2 ' for the middle layer potential.

Finally, the existence of a line source at the point $\left(x_{s}, y_{s}\right)$, requires that

$$
\phi_{2}(x, y) \rightarrow-\frac{\mu}{2 \pi} \ln \left[\left(x-x_{s}\right)^{2}+\left(y-y_{s}\right)^{2}\right]^{1 / 2} \quad \text { as }(x, y) \rightarrow\left(x_{s}, y_{s}\right)
$$

\section{$3 \quad$ Integral equation for two free surfaces}

A Green's function approach similar to that used by Hocking and Forbes [2] is used. We seek a function $\mathrm{G}$ that satisfies (13) subject to the condition (14):

$$
\begin{array}{ll} 
& \nabla^{2} G=\delta\left(x-x_{0}, y-y_{0}\right), \\
\text { with } \quad & G\left( \pm \frac{\alpha}{2}, y ; x_{0}, y_{0}\right)=0, \quad-\infty<y<\infty
\end{array}
$$

where $\delta\left(x-x_{0}, y-y_{0}\right)$ is the Dirac delta function. 
These conditions set $G=0$ at $x= \pm \alpha / 2$, along the seepage faces. Using conformal mapping techniques, a suitable form for $\mathrm{G}$ is

$$
G=\frac{1}{4 \pi} \ln \left[\left(f-f_{0}\right)^{2}+\left(g-g_{0}\right)^{2}\right]-\frac{1}{4 \pi} \ln \left[\left(f-f_{0}\right)^{2}+\left(g+g_{0}\right)^{2}\right],
$$

and we determine that

$$
\begin{aligned}
& G_{x}=\frac{1}{2 \alpha}\left[\frac{\left(f-f_{0}\right) g-\left(g-g_{0}\right) f}{\left(f-f_{0}^{2}+\left(g-g_{0}\right)^{2}\right.}-\frac{\left(f-f_{0}\right) g-\left(g+g_{0}\right) f}{\left(f-f_{0}\right)^{2}+\left(g+g_{0}\right)^{2}}\right], \\
& G_{y}=\frac{1}{2 \alpha}\left[\frac{\left(f-f_{0}\right) f+\left(g+g_{0}\right) g}{\left(f-f_{0}\right)^{2}+\left(g+g_{0}\right)^{2}}-\frac{\left(f-f_{0}\right) f+\left(g-g_{0}\right) g}{\left(f-f_{0}\right)^{2}+\left(g-g_{0}\right)^{2}}\right],
\end{aligned}
$$

where $f=f(x, y), g=g(x, y), f_{0}=f\left(x_{0}, y_{0}\right)$ and $g_{0}=g\left(x_{0}, y_{0}\right)$, and

$$
\begin{aligned}
f(x, y) & =e^{-\pi y / \alpha} \sin \frac{\pi x}{\alpha}, \\
g(x, y) & =e^{-\pi y / \alpha} \cos \frac{\pi x}{\alpha}, \\
f_{x}(x, y) & =\frac{\pi}{\alpha} e^{-\pi y / \alpha} \cos \frac{\pi x}{\alpha}=\frac{\pi}{\alpha} g \\
f_{y}(x, y) & =-\frac{\pi}{\alpha} e^{-\pi y / \alpha} \sin \frac{\pi x}{\alpha}=-\frac{\pi}{\alpha} f \\
g_{x}(x, y) & =-\frac{\pi}{\alpha} e^{-\pi y / \alpha} \sin \frac{\pi x}{\alpha}=-\frac{\pi}{\alpha} f \\
g_{y}(x, y) & =-\frac{\pi}{\alpha} e^{-\pi y / \alpha} \cos \frac{\pi x}{\alpha}=-\frac{\pi}{\alpha} g .
\end{aligned}
$$

Now consider Green's second identity

$$
\iint_{A}\left(\phi \nabla^{2} G-G \nabla^{2} \phi\right) d A=\int_{\Gamma}\left(\phi \frac{\partial G}{\partial \vec{n}}-G \frac{\partial \phi}{\partial \vec{n}}\right) d S
$$

where $A$ refers to the interior of the domain bounded by the upper and lower interfaces and the two seepage faces, denoted as $\Gamma$; see Figure 2. Noting that $\nabla^{2} \phi=0$ everywhere except at $\left(x_{s}, y_{s}\right)$, the source location, and that $\mathrm{G}$ satisfies Laplace's equation, that is $\nabla^{2} \mathrm{G}=0$ except at $\left(\mathrm{x}_{0}, \mathrm{y}_{0}\right)$, and that 


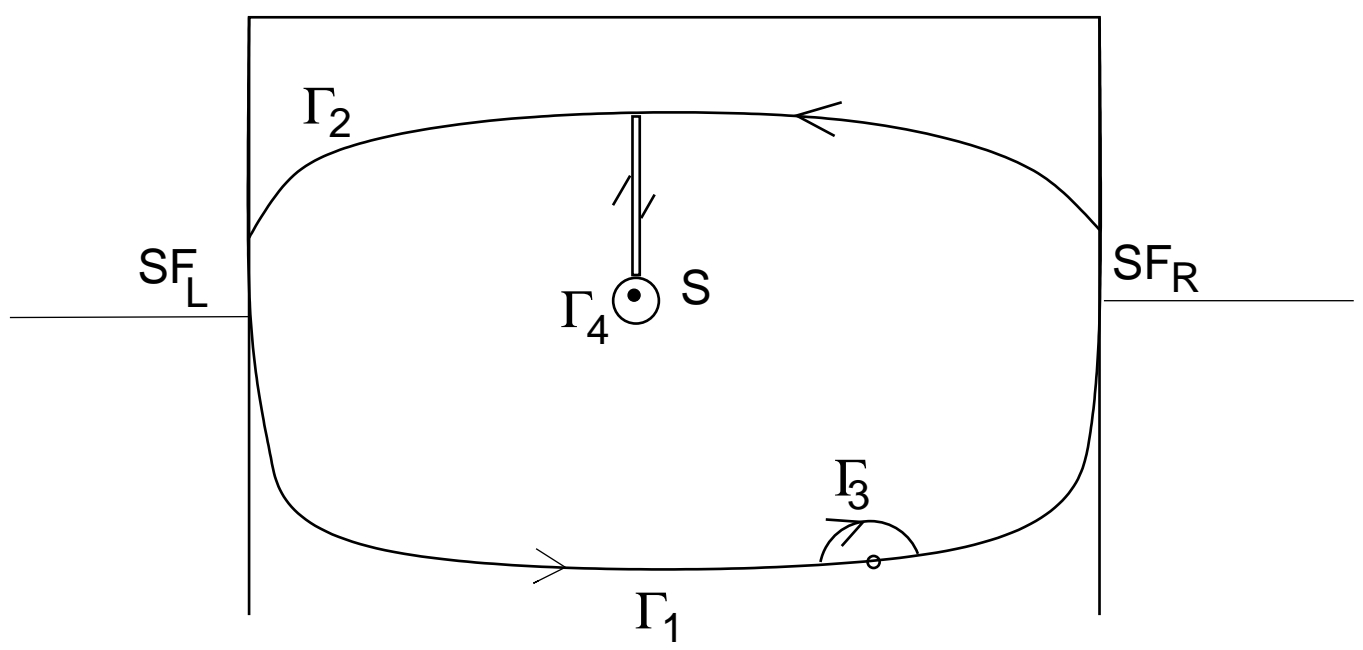

Figure 2: Schematic diagram of island of length $\alpha$ and the integration paths $\Gamma_{1}, \Gamma_{2}, \Gamma_{3}, \Gamma_{4}$ and the seepage faces $S F_{L}$ and $S F_{R} . \Gamma_{3}$ goes around the point $\left(x_{0}, y_{0}\right)$, while $\Gamma_{4}$ goes around the source point $\left(x_{s}, y_{s}\right)$.

$\mathrm{G}=0$ along the seepage face, the left hand side of the identity is zero if the path of integration omits $\left(x_{0}, y_{0}\right)$ and $\left(x_{s}, y_{s}\right)$. Therefore, if we choose the path of integration to be around the boundary but with small circles that exclude the singularities at $\left(x_{0}, y_{0}\right)$ and $\left(x_{s}, y_{s}\right)$ as shown in Figure 2, we are left with an integral equation for integration around the boundary of region 2 only.

After careful substitution of the boundary conditions where $\partial \phi / \partial \vec{n}=0$ and $\mathrm{G}=0$ as appropriate, adding and subtracting a term to remove the singularity as $(x, y) \rightarrow\left(x_{0}, y_{0}\right)$, and carefully integrating around the two loops $\Gamma_{3}$ and $\Gamma_{4}$, we are left with

$$
\begin{aligned}
& \int_{-\alpha / 2}^{\alpha / 2}\left(\phi-\phi_{0}\right)\left[\eta_{1}^{\prime} G_{x}-G_{y}\right] d x+\int_{-\alpha / 2}^{\alpha / 2}\left(\phi-\phi_{0}\right)\left[\eta_{2}^{\prime} G_{x}-G_{y}\right] d x \\
& -\frac{\mu}{4 \pi} \ln \left[\frac{\left(f_{s}-f_{0}\right)^{2}+\left(g_{s}-g_{0}\right)^{2}}{\left(f_{s}-f_{0}\right)^{2}+\left(g_{s}+g_{0}\right)^{2}}\right]+I_{1}+I_{2}=0,
\end{aligned}
$$


where $I_{1}$ and $I_{2}$ correspond to integrals along the seepage faces,

$$
\begin{aligned}
& \mathrm{I}_{1}=\frac{1}{\alpha} \int_{0}^{\beta_{+}}\left(\phi-\phi_{0}\right)\left[\frac{e^{-\pi y / \alpha}\left(e^{-\pi y_{0} / \alpha} \cos \frac{\pi x_{0}}{\alpha}\right)}{\left(e^{-\pi y / \alpha}-e^{-\pi y_{0} / \alpha}\right)^{2}+\left(e^{-\pi y_{0} / \alpha} \cos \frac{\pi x_{0}}{\alpha}\right)^{2}}\right] d y, \\
& I_{2}=\frac{1}{\alpha} \int_{0}^{\beta_{-}}\left(\phi-\phi_{0}\right)\left[\frac{e^{-\pi y / \alpha}\left(e^{-\pi y_{0} / \alpha} \cos \frac{\pi x_{0}}{\alpha}\right)}{\left(e^{-\pi y / \alpha}+e^{-\pi y_{0} / \alpha}\right)^{2}+\left(e^{-\pi y_{0} / \alpha} \cos \frac{\pi x_{0}}{\alpha}\right)^{2}}\right] d y,
\end{aligned}
$$

and $\phi_{0}=\phi\left(x_{0}, y_{0}\right)$ which, combined with (9) and (10) provides a closed system for the unknown locations of the two interfaces, $\eta_{1}(x)$ and $\eta_{2}(x)$. The recharge source is placed in the horizontal center of the island so that all of the interfaces calculated will be symmetric.

\section{Numerical method}

The integral equation (25) is highly nonlinear and therefore we adopt a numerical approach. A discrete approximation is found by taking points $x_{j}=x_{1}, x_{2}, \ldots, x_{n}$ in $x$ with the aim to find $\phi_{j}, \eta_{1_{j}}$ and $\eta_{2_{j}}$ for $j=1,2, \ldots, n$. This gives rise to $2 n$ equations in $2 n$ unknowns, each corresponding to a point in the discrete representation of $-\alpha / 2<x<\alpha / 2$. The discretised equation is

$$
\begin{aligned}
& \sum_{j=0}^{n}\left(\phi_{j}-\phi_{i}\right)\left[\eta_{1}^{\prime}\left(x_{j}\right) G_{x_{j}}-G_{y_{j}}\right] \Delta x_{j} w_{j} \\
& +\sum_{j=0}^{n}\left(\phi_{n+j}-\phi_{i}\right)\left[\eta_{2}^{\prime}\left(x_{j}\right) G_{x_{j}}-G_{y_{j}}\right] \Delta x_{j} w_{j} \\
& -\frac{\mu}{4 \pi} \ln \left[\frac{\left(f_{s_{j}}-f_{i}\right)^{2}+\left(g_{s_{j}}-g_{i}\right)^{2}}{\left(f_{s_{j}}-f_{i}\right)^{2}+\left(g_{s_{j}}+g_{i}\right)^{2}}\right]+\frac{1}{\alpha}\left(I_{1}+I_{2}\right) \\
& =0, \quad \text { for } i=1,2, \ldots, 2 n,
\end{aligned}
$$


where $\Delta x_{j}=x_{j+1}-x_{j}$ is the step size and $w_{j}$ is the weighting for the trapezoidal rule. $\mathrm{I}_{1}$ and $\mathrm{I}_{2}$ are as in equations (26) and are accurately evaluated using standard techniques.

The values of $\phi_{i}$ correspond to those on both surfaces, and are replaced using conditions (9) and (10), leaving the unknown interface locations $\eta_{1 i}$ and $\eta_{2 i}$, $i=1,2, \ldots, n$, as the $2 n$ unknowns. The end points of the lower interface are known to lie at $(-\alpha / 2,0)$ and $(\alpha / 2,0)$ and so these are omitted from the scheme, leaving $2 n-2$ nonlinear equations in $2 n-2$ unknowns.

In (25) there is a possible singularity in the integrand as $(x, \eta) \rightarrow\left(x_{0}, \eta_{0}\right)$. Carefully taking the limit in the two integrals as $(x, y) \rightarrow\left(x_{0}, y_{0}\right)$ gives

$$
\lim _{x \rightarrow x_{0}}\left(\phi-\phi_{0}\right)\left[\eta_{k}^{\prime}(x) G_{x}-G_{y}\right]=\frac{1}{2 \pi}\left(1-\gamma_{k}\right)\left[\eta_{k}^{\prime}(x)\right]^{2}, \quad k=1,2 .
$$

These values were incorporated into the trapezoidal integration scheme.

A damped Newton's method solved the system of $2 n-2$ nonlinear equations. Thus, an initial guess for the values of $\eta_{1}\left(x_{i}\right), i=2, \ldots n-1$, and $\eta_{2}\left(x_{i}\right)$, $i=1,2, \ldots, n$, was updated iteratively. Most simulations were performed with a space step of $\Delta x=0.3$ and this gave graphical accuracy for the interfaces and seepage face heights.

\section{$5 \quad$ Results}

Simulations were carried out for different inflow rates, $\mu$, with $\gamma_{1}=1.5$ and $\gamma_{1}=1.1$, both with $\gamma_{2}=0$ (air-water) for several different island lengths and source locations. For a given value of $\mu$ and $\gamma_{1}$, there is a corresponding seepage face height $\beta$ to which the numerical scheme will converge.

Figure 3 shows interface shapes for the case of an island of length $\alpha=50$ where the recharge source is located at $\left(x_{s}, y_{s}\right)=(0,0)$ with $\gamma_{1}=1.5$ and $\gamma_{2}=0$. As the pumping rate $\mu$ increases, the height (and depth) of the two 


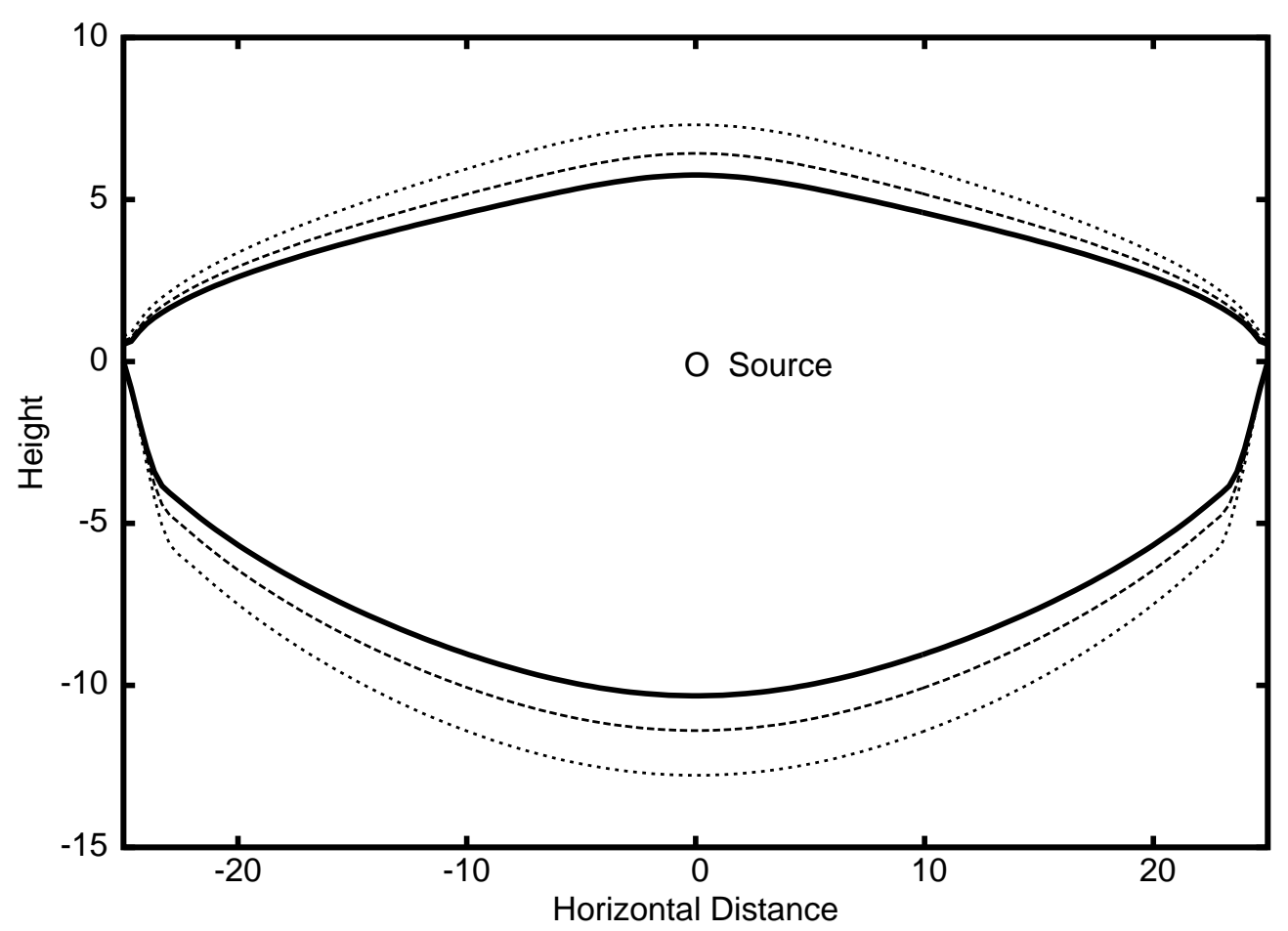

Figure 3: Typical interface shapes for an island of width $\alpha=50$ with $\mu=4$ (solid), $\mu=5$ (dash), $\mu=6.5$ (dots) for density ratios $\gamma_{1}=1.5$ and $\gamma_{2}=0$. 


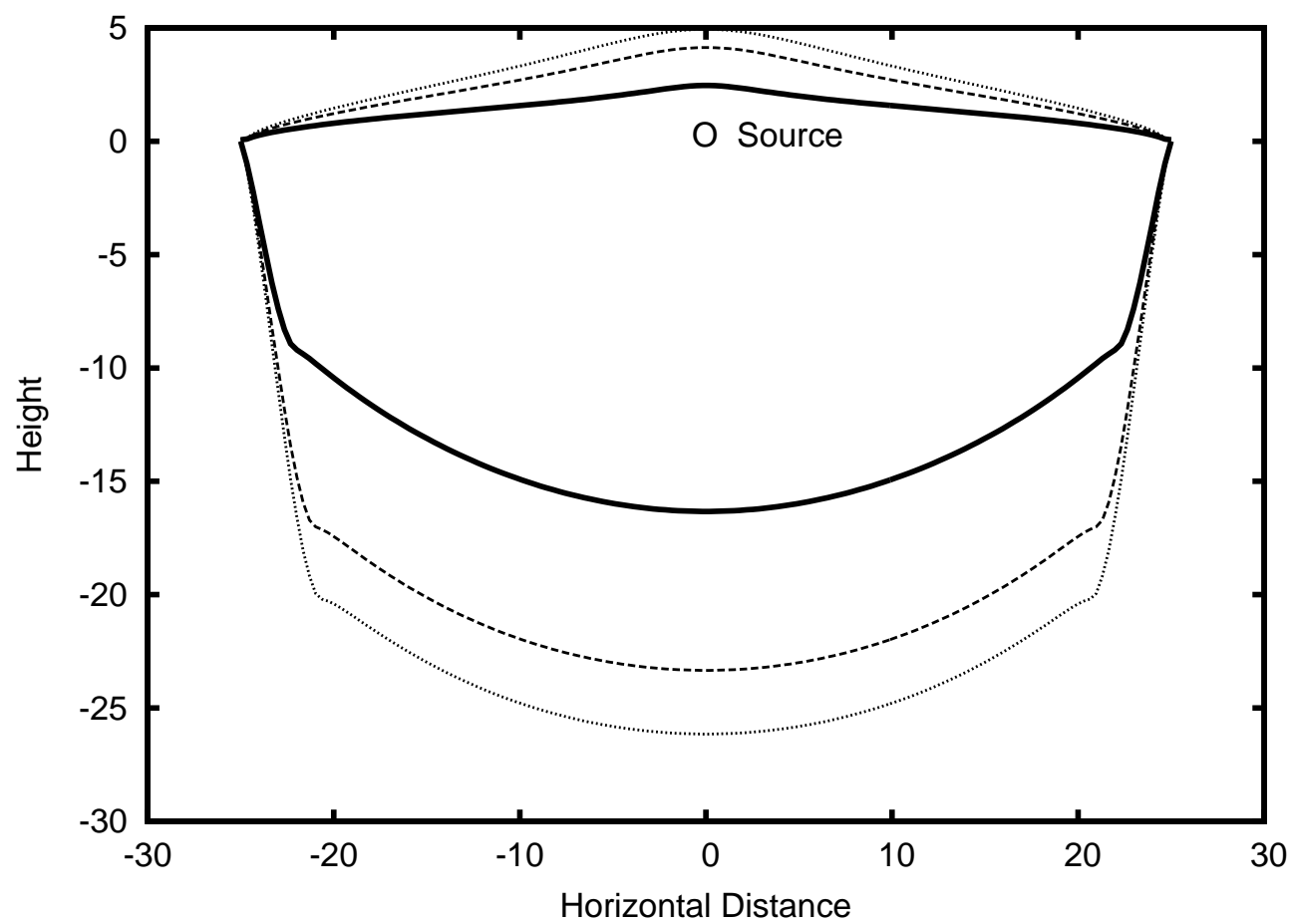

Figure 4: Typical interface shapes for an island of width $\alpha=50$ with $\mu=1.65$ (solid), $\mu=4$ (dash), $\mu=5.6$ (dots) for density ratios $\gamma_{1}=1.1$ and $\gamma_{2}=0$.

interfaces increases almost linearly. There is a particular window of pumping rates that has a stable steady solution. In this case, $\mu$ ranges from about $\mu=3.7$ up to $\mu=6.5$.

Figure 4 shows interface shapes for the case of an island of length $\alpha=50$ where the recharge source is located at $\left(x_{s}, y_{s}\right)=(0,0)$ and $\gamma_{1}=1.1$ with $\gamma_{2}=0$. Notice that the seepage face is much smaller in this example and the depth of the lower interface is much greater. This agrees with the results of Polubarinova-Kochina [7] who used approximate methods to show that 


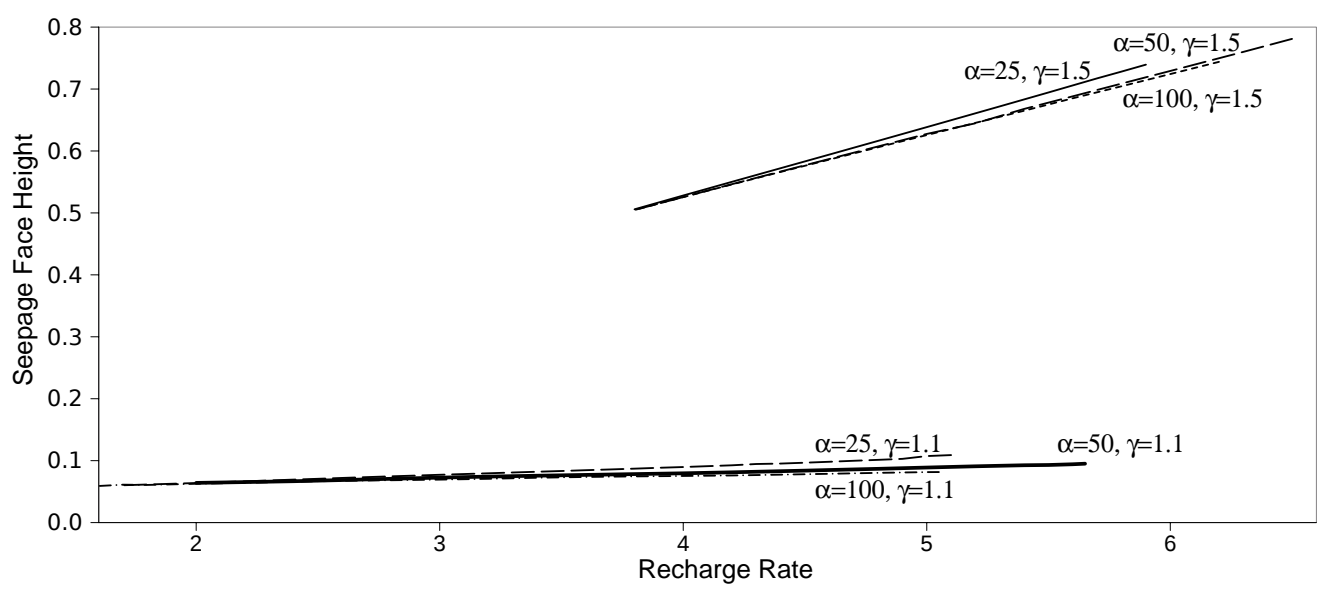

FiguRE 5: Variation in seepage face heights with flow rate for an island of width $\alpha=25,50,100$ for density ratios $\gamma_{1}=1.5$ and $\gamma_{1}=1.1$. The relationship is clearly linear and almost unaffected by island length.

$\eta_{1}(x) \propto 1 /\left(1-\gamma_{1}\right)$. Again, there is a particular window of pumping rates over which there is a stable steady solution. In this case, $\mu$ ranges from about $\mu=1.65$ up to $\mu=5.6$.

There is no obvious physical reason in the solutions that suggests why flow rates are limited in this way. Stagnation points exist on the interfaces both above and below the source at all flow values, but on neither surface is there extreme behaviour as the limits are approached. The cases shown in Figure 4 show a slight concavity of the upper surface as the flow rate increases, but this does not appear in the case shown in Figure 3. The lower interface seems to have quite a sharp corner near the ends of the island before levelling off, and this point may warrant further investigation as a possible cause of the limiting behaviour.

In both cases, increasing the flow rate increases the height of the seepage face, albeit slowly. Figure 5 shows this variation for the case $\alpha=25,50,100$ 
for varying flow rates $\mu$ with $\gamma_{1}=1.5$ and $\gamma_{1}=1.1$. Clearly, the different density ratios give very different seepage face heights. However, the effect of the nondimensional island width, $\alpha$, on the value of the seepage face height, $\beta$, appears to be quite small, and even the recharge rate, $\mu$, has only a small effect. This is not surprising since the flux through the ends of the island for a given flow rate is identical, irrespective of the island length. In fact, there is a very minor increase in seepage face height as the island gets longer. Moving the recharge point up or down was found to have very little impact on the shape of the interfaces or the seepage face heights.

\section{Concluding remarks}

We developed a method to solve for the interfaces of the lens of freshwater beneath an island. No recharge is allowed through the surface of the lens, and artificial recharge of water is through a single source. No withdrawal was included, but the method is adaptable enough to include it. In order to obtain a steady state, seepage faces are allowed at both ends of the island. In the work presented here, only one source is allowed and it is located centrally (in the horizontal direction), giving left-right symmetric surfaces. The height of the upper interface suggests that for low lying islands the upper interface would probably break the surface in many cases, and most of the island would indeed be saturated, as considered by Hocking and Forbes [2].

The work differs from the previous work by allowing a second interface. A steady, stable lens can be maintained using a single recharge point, and the resulting seepage face heights are almost totally dominated by the density ratios between the fluid layers. There are limits on the recharge that will produce a steady solution. Further work will compare different locations for the source and also include several withdrawal points to optimise the water retention. 


\section{References}

[1] L. K. Forbes, G. C. Hocking and S. Wotherspoon, 2004, Salt-water up-coning during extraction of fresh water from a tropical island, $J$. Eng. Math, 48, 2004, 69-91. doi:10.1023/B:ENGI.0000009517.28682.6f $\mathrm{C} 2$

[2] G. C. Hocking and L. K. Forbes, The lens of freshwater in a tropical island - 2d withdrawal, Comp. \& Fluids, 33, 2004, 19-30. doi:10.1016/S0045-7930(03)00035-5 C2, C5, C13

[3] G. C. Hocking and H. Zhang, A note on withdrawal from a two-layer fluid through a line sink in a porous medium, ANZIAM J., 50, 2008, 101-110. doi:10.1017/S144618110800028X C2

[4] C. D. Langevin, M. T. Stewart and C. M. Beaudoin, Effects of sea water canals on fresh water resources: an example from Big Pine Key, Florida, Ground Water, 36, 1998, 503-513. doi:10.1111/j.1745-6584.1998.tb02822.x C2

[5] S. K. Lucas, J. R. Blake and A. Kucera, A boundary-integral method applied to water coning in oil reservoirs, J. Austral. Math. Soc. Ser. B, 32, 1991, 261-283. doi:10.1017/S0334270000006858 C2

[6] M. Muskat and R. B. Wyckoff, An approximate theory of water coning in oil production, Trans. AIME, 114, 1935, 144-163. C2

[7] P. Y. Polubarinova-Kochina, Unsteady seepage with an interface (in Russian), Moscow: Dokl. Nauk, S. S. S. R., 1949, 173-176 C11

[8] C. Ruppel, G. Schultz and S. Kruse, Anomalous fresh water lens morphology on a strip barrier island, Ground Water, 38, 2000, 872-881. doi:10.1111/j.1745-6584.2000.tb00686.x C2 
[9] H. Zhang and G. C. Hocking, Withdrawal of layered fluid through a line sink in a porous medium, J. Austral. Math. Soc. Ser. B, 38, 1996, 240-254. doi:10.1017/S0334270000000631 C2

\section{Author addresses}

1. S. A. Chen, Mathematics and Statistics, Murdoch University Murdoch, WA 6150, Australia.

2. G. C. Hocking, Mathematics and Statistics, Murdoch University Murdoch, WA 6150, Australia.

mailto:G.Hocking@murdoch.edu . au 